\title{
Sensibilidade "in vitro" ao benomyl por isolados de Colletotrichum spp. associados à mancha da gala em macieira
}

\author{
Natasha Akemi Hamada ${ }^{1}$, Yoshinori Katsurayama² \& Adriana C.M. Dantas ${ }^{3}$ \\ ${ }^{1}$ Centro Universitário Católico do Sudoeste do Paraná, 85.555-000, Palmas, PR, Brasil; ${ }^{2}$ Epagri - Estação Experimental \\ de São Joaquim, 88.600-000, São Joaquim, SC, Brasil; ${ }^{3}$ Universidade Estadual do Rio Grande do Sul, 95.700-000, Bento \\ Gonçalves, RS, Brasil
}

Autor para correspondência: Natasha A. Hamada, e-mail: natiakemi23@hotmail.com

\section{RESUMO}

Colletotrichum gloeosporioides e C. acutatum são os agentes etiológicos da Mancha da Gala (MG), na cultura da macieira, no Brasil. Programas de melhoramento visando o desenvolvimento de resistência genética à doença necessitam do conhecimento pleno das características das espécies do patógeno envolvidas. Uma maneira de separar estas espécies é através do estudo da sensibilidade de isolados ao fungicida benomyl. Através do teste de sensibilidade de 39 isolados de C. acutatum e C. gloeosporioides relacionados à MG, em que foram avaliados a taxa de crescimento micelial e o índice de redução deste crescimento, observou-se que dentre os isolados de C. gloeosporioides há um grupo resistente ao fungicida e que difere de todos os isolados desta espécie relatados neste patossistema. Este grupo mostrou-se resistente às concentrações de 5 e $100 \mu \mathrm{g} \cdot \mathrm{mL}^{-1}$ com índice de redução (IR) variando entre $0 \%$ e $8 \%$, e entre 15 e $30 \%$, respectivamente, enquanto o isolado padrão (CG197) apresentou IR de 100\% em ambas as concentrações testadas. Os isolados de $C$. acutatum foram todos resistentes ao fungicida. Devido à resistência de alguns isolados de C. gloeosporioides, a separação de espécies de Colletotrichum associadas à macieira, baseada apenas na resistência ou suscetibilidade ao fungicida benomyl, não deve ser utilizada isoladamente.

Palavras-chave: Malus spp., resistência a fungicida, crescimento in vitro.

\begin{abstract}
In vitro assessment of sensitivity to benomyl of Colletotrichum spp. isolates associated with Gala leaf spot of apple

In Brazil, Colletotrichum gloeosporioides and C. acutatum are responsible for the occurrence of Gala leaf spot or Glomerella Leaf Spot (GLS) on apple. Breeding programs aiming to develop genetic resistance to disease need a complete understanding of the characteristics of involved species. A study of the sensibility of the isolates to benomyl is a way to separate those species. A sensitivity test of 39 isolates of $C$. acutatum and C. gloeosporioides involved with GLS, evaluated by growth rate and reduction rate of mycelium, revealed a group of $C$. gloeosporioides isolates that are resistant to the fungicide. This group showed resistance to concentrations of 5 and $100 \mu \mathrm{g} \cdot \mathrm{mL}^{-1}$ with reduction rate varying between $0 \%$ and $8 \%$, and between 15 and $30 \%$, respectively. While the standard $C$. gloeosporioides isolate (CG197) showed a reduction rate of $100 \%$ at both tested concentrations, all isolates of C. acutatum were resistant to the fungicide. Due to the resistance of some isolates of C. gloeosporioides, the separation of Colletotrichum species on apple should not be based only on resistance or susceptibility to benomyl.
\end{abstract}

Keywords: Malus spp., fungicide resistance, in vitro growth.

Desde o seu primeiro relato, em 1983, no município de Porto Amazonas, PR (Leite et al., 1988), a Mancha da Gala (ou Mancha foliar da Gala ou Mancha foliar de Glomerella) vem se espalhando por todos os pomares de maçã do sul do Brasil. A doença ficou restrita ao Estado do Paraná durante aproximadamente seis anos e, então, a partir do ciclo 1989/90, se disseminou pelos pomares de

Parte da Dissertação de Mestrado do primeiro autor. Universidade Federal de Santa Catarina. Florianópolis SC. 2006. maçã de Fraiburgo SC, Vacaria RS, e São Joaquim SC, em 1993. A doença se manifesta durante o verão, causando desfolhamento severo das macieiras, provocando lesões avermelhadas nas folhas e pequenas pontuações deprimidas nos frutos (Boneti et al., 2002).

É relatado que três espécies de Colletotrichum estão associadas à Mancha da Gala, sendo elas Colletotrichum acutatum, C. gloeosporioides e uma espécie ainda não identificada, sendo as duas últimas mais agressivas (Katsurayama et al., 2000). Katsurayama et al. (2001) relataram que isolados de Colletotrichum podem causar 
sintomas diferentes de MG em macieira, aos quais chamaram de Mancha necrótica.

A sensibilidade de isolados de Colletotrichum ao fungicida benomyl, do grupo dos benzimidazóis, embora tenha sido inicialmente utilizada apenas para estimar o potencial de controle químico do composto sobre o gênero, pode ser utilizada como critério de diferenciação entre espécies de Colletotrichum (Freeman et al., 1998). A sensibilidade ao benomyl tem sido utilizada em vários trabalhos de diferenciação de isolados, como o desenvolvido por Shabi et al. (1993) que distinguiram isolados de $C$. gloeosporioides oriundos de diferentes hospedeiros em Israel; e o de Peres et al. (2004) que utilizaram esse critério para separar isolados de C. acutatum e C. gloeosporioides em Citrus, entre outros.

A diferenciação entre espécies fitopatogênicas de Colletotrichum é importante para a implementação de eficientes estratégias de controle, as quais serão úteis em programas de melhoramento visando à caracterização da resistência ao Colletotrichum spp. (Chung et al., 2006). O objetivo deste trabalho foi estudar a viabilidade do uso do fungicida benomyl na diferenciação das espécies de Colletotrichum relacionadas à Mancha da Gala, na cultura da macieira.

Foram utilizados 39 isolados monospóricos préidentificados como sendo da espécie $C$. gloeosporioides (69\%) ou C. acutatum (31\%), oriundos de folhas e frutos de macieira, cv. Gala, provenientes de diversos pomares produtores (Tabela 1). A identificação dos isolados foi realizada com base nas características das espécies de $C$. gloeosporioides e C. acutatum descritas por Boneti et al. (1999b). Estes isolados foram selecionados por apresentarem boa produção de esporos em meio de cultura e características típicas (cor, forma, velocidade de crescimento) in vitro das espécies estudadas. Foram utilizadas placas de Petri $\varnothing$ $=90 \mathrm{~mm}$ ) contendo meio BDA suplementado com três diferentes concentrações do fungicida $\left(0,5\right.$ e $\left.100 \mu \mathrm{g} \cdot \mathrm{mL}^{-1}\right)$. Diariamente, durante 6 dias, foram medidos o diâmetro das colônias ( $\mathrm{mm}$ ) e a partir deste calculada a taxa de crescimento (TC) de cada um dos isolados. A TC foi calculada através da fórmula $\mathrm{TC}=\left(\mathrm{D}_{\mathrm{n}}-\mathrm{a}\right) / \mathrm{n}$, onde $\mathrm{D}_{\mathrm{n}}=$ diâmetro da colônia $\mathrm{n}$ dias após a transferência, $\mathrm{a}=$ diâmetro do disco micelial inicial e $\mathrm{n}=$ número de dias de incubação (Teixeira et al., 2001). Os isolados que não cresceram na presença do fungicida, diferindo entre os tratamentos que continham o benomyl, foram considerados sensíveis; os isolados que cresceram em ambas as concentrações testadas e que não diferiram entre elas foram considerados resistentes.

Os isolados CG197 e CA 35, da Epagri - Estação Experimental de São Joaquim foram utilizados como padrão de C. gloeosporioides e C. acutatum, respectivamente. Com base no crescimento micelial destes isolados foi calculado o índice de redução do crescimento micelial dos demais (IR\%), que estabelece a relação percentual entre o crescimento de cada isolado em relação aos padrões. O delineamento experimental utilizado foi o inteiramente casualizado, com três repetições para cada isolado, por concentração testada.
Os dados obtidos nos ensaios foram submetidos à análise multivariada e posterior teste de agrupamento pelo método de Ward.

A análise multivariada dos dados da taxa de crescimento dos isolados permitiu separá-los em quatro grupos distintos, de acordo com a concentração de benomyl a que estes foram submetidos (Figura 1). Todos os isolados de C. acutatum mostraram-se resistentes ao benomyl quando comparados ao isolado padrão CA35. Os IR\% dos isolados de C. acutatum trabalhados variaram entre 47 e $76 \%\left(5 \mu \mathrm{g} \cdot \mathrm{mL}^{-1}\right)$ e entre 56 e $78 \%(100 \mu \mathrm{g} \cdot \mathrm{mL}$ $\left.{ }^{1}\right)$, respectivamente. O isolado padrão (CA35) apresentou IR $\%$ de 48 e $73 \%$ para as concentrações de $5 \mu \mathrm{g} \cdot \mathrm{mL}^{-1} \mathrm{e}$ $100 \mu \mathrm{g} \cdot \mathrm{mL}^{-1}$, respectivamente, comparados ao tratamento testemunha (sem adição de benomyl).

Todos os isolados de C. acutatum mostraram-se resistentes ao fungicida testado, à semelhança do que foi observado por Boneti et al. (1999a) através do levantamento de espécies envolvidas com a MG no Estado de Santa Catarina, e por Chung et al. (2006) em trabalho com espécies de Colletotrichum provenientes de várias frutas, dentre elas a maçã, no Japão. A baixa sensibilidade observada nas espécies de C. acutatum ao fungicida benomyl vem sendo utilizada como critério de classificação de espécies em hospedeiros como pessegueiro e macieira (Berstein et al., 1995; Boneti et al., 1999b), morango, abóbora, melão, ciclâmen (Ishii et al., 1998) e em Citrus (Brown et al., 1996; Peres et al., 2004). Esta sensibilidade moderada ou baixa (observada nos isolados aqui classificados como resistentes) de isolados de C. acutatum já foi relatada na literatura por Goes et al. (1998) em isolados oriundos de frutos de Citrus, que tiveram redução de 40 e $85 \%$ no crescimento micelial quando foram submetidos a concentrações de benomyl de 1 e $2,5 \mu \mathrm{g} . \mathrm{mL}^{-1}$, respectivamente.

Em relação aos isolados de C. gloeosporioides (69\%), a maioria deles mostrou-se sensível ao fungicida testado, exceção feita a cinco isolados $(18,5 \%)$ provenientes de um único pomar comercial localizado no município de Campo Tenente PR. Dentre os isolados resistentes, estes foram os que apresentaram a maior taxa de crescimento micelial durante todo o período do ensaio, em ambas as concentrações testadas, com médias variando de 0,26 a 0,27 $\mathrm{mm}\left(5 \mu \mathrm{g} \cdot \mathrm{mL}^{-1}\right)$ e de 0,27 a $0,28 \mathrm{~mm}\left(100 \mu \mathrm{g} \cdot \mathrm{mL}^{-1}\right)$ (Tabela 1). O IR\% destes isolados na concentração de $5 \mu \mathrm{g} \cdot \mathrm{mL}^{-1}$ variou entre $0 \%$ e $8 \%$, e entre 15 e $30 \%$ na concentração de $100 \mu \mathrm{g} \cdot \mathrm{mL}^{-1}$. Nestas concentrações o isolado padrão (CG197) apresentou IR\% de 100\%, ou seja, em relação ao padrão os isolados provenientes do pomar de Campo Tenente/ PR praticamente não sofreram redução no seu crescimento micelial quando submetidos ao benomyl. Estas observações contestam a maioria das encontradas na literatura, como por exemplo a de Freeman et al. (1998), que afirmam que isolados desta espécie são altamente sensíveis ao benomyl, pois não apresentaram nenhum tipo de crescimento na presença deste. Entretanto, Peres (2004) afirma que a causa da baixa sensibilidade desta espécie ao 
TABELA 1 - Numeração, origem geográfica, origem vegetal, espécie, resistência ao benomyl e taxa de crescimento micelial (mm/dia), dos isolados de Colletotrichum utilizados no presente trabalho

\begin{tabular}{|c|c|c|c|c|c|c|c|}
\hline \multirow[t]{3}{*}{ Isolado } & \multirow[t]{3}{*}{ Procedência } & \multirow[t]{3}{*}{$\begin{array}{l}\text { Órgão de isolamento } \\
\text { / Tipo de sintoma }\end{array}$} & \multirow[t]{3}{*}{ Espécie $^{2}$} & \multirow[t]{3}{*}{$\begin{array}{c}\text { Resistência ao } \\
\text { benomyl }\end{array}$} & \multirow{2}{*}{\multicolumn{3}{|c|}{$\begin{array}{c}\text { Taxa de crescimento } \\
\text { micelial }(\mathrm{mm} / \mathrm{dia})\end{array}$}} \\
\hline & & & & & & & \\
\hline & & & & & $\mathbf{0}$ & 5 & 100 \\
\hline 1 & Caçador/ SC & $\mathrm{Fl} / \mathrm{MG}$ & $\mathrm{Ca}$ & Resistente & 0,26 & 0,17 & 0,18 \\
\hline 2 & Caçador/ SC & $\mathrm{Fl} / \mathrm{MG}$ & $\mathrm{Ca}$ & Resistente & 0,27 & 0,18 & 0,16 \\
\hline 3 & Caçador/ SC & $\mathrm{Fl} / \mathrm{MG}$ & $C g$ & Sensível & 0,28 & 0,00 & 0,00 \\
\hline 4 & Vacaria/ RS & $\mathrm{Fl} / \mathrm{MG}$ & $C g$ & Sensível & 0,24 & 0,00 & 0,00 \\
\hline 5 & Vacaria/ RS & $\mathrm{Fl} / \mathrm{MG}$ & $\mathrm{Cg}$ & Sensível & 0,23 & 0,00 & 0,00 \\
\hline 6 & Frei Rogério/ SC & $\mathrm{Fl} / \mathrm{MG}$ & $C g$ & Sensível & 0,23 & 0,00 & 0,00 \\
\hline 7 & Frei Rogério/ SC & $\mathrm{Fl} / \mathrm{MG}$ & $\mathrm{Cg}$ & Mod. Sens. & 0,23 & 0,00 & 0,00 \\
\hline 8 & Frei Rogério/ SC & $\mathrm{Fl} / \mathrm{MG}$ & $\mathrm{Cg}$ & Mod. Sens. & 0,23 & 0,00 & 0,00 \\
\hline 9 & Frei Rogério/ SC & $\mathrm{Fl} / \mathrm{MG}$ & $\mathrm{Cg}$ & Sensível & 0,27 & 0,00 & 0,00 \\
\hline 10 & Curitibanos/ SC & $\mathrm{Fl} / \mathrm{MG}$ & $C g$ & Sensível & 0,25 & 0,00 & 0,00 \\
\hline 11 & São Joaquim/ SC & $\mathrm{Fl} / \mathrm{MG}$ & $C g$ & Sensível & 0,24 & 0,00 & 0,00 \\
\hline 12 & São Joaquim/ SC & $\mathrm{Fl} / \mathrm{MG}$ & $\mathrm{Cg}$ & Sensível & 0,25 & 0,00 & 0,00 \\
\hline 13 & São Joaquim/ SC & $\mathrm{Fl} / \mathrm{MG}$ & $\mathrm{Cg}$ & Sensível & 0,25 & 0,00 & 0,00 \\
\hline 14 & Palmas/ PR & $\mathrm{Fl} / \mathrm{MG}$ & $C g$ & Sensível & 0,23 & 0,00 & 0,00 \\
\hline 15 & Fraiburgo/ SC & $\mathrm{Fl} / \mathrm{MG}$ & $\mathrm{Cg}$ & Sensível & 0,27 & 0,00 & 0,00 \\
\hline 16 & Fraiburgo/ SC & $\mathrm{Fl} / \mathrm{MG}$ & $\mathrm{Cg}$ & Sensível & 0,27 & 0,00 & 0,00 \\
\hline 17 & Campo Tenente/ PR & $\mathrm{Fl} / \mathrm{MG}$ & $\mathrm{Cg}$ & Resistente & 0,28 & 0,26 & 0,27 \\
\hline 18 & Campo Tenente/ PR & $\mathrm{Fl} / \mathrm{MG}$ & $\mathrm{Cg}$ & Resistente & 0,27 & 0,27 & 0,28 \\
\hline 19 & Campo Tenente/ PR & $\mathrm{Fl} / \mathrm{MG}$ & $\mathrm{Cg}$ & Resistente & 0,27 & 0,26 & 0,28 \\
\hline 20 & Campo Tenente/ PR & $\mathrm{Fl} / \mathrm{MG}$ & $\mathrm{Cg}$ & Resistente & 0,27 & 0,27 & 0,28 \\
\hline 21 & Campo Tenente/ PR & $\mathrm{Fl} / \mathrm{MG}$ & $\mathrm{Cg}$ & Resistente & 0,28 & 0,27 & 0,27 \\
\hline 22 & Palmeira/ PR & $\mathrm{Fl} / \mathrm{MG}$ & $\mathrm{Cg}$ & Sensível & 0,28 & 0,00 & 0,00 \\
\hline 23 & Palmeira/ PR & $\mathrm{Fl} / \mathrm{MG}$ & nd & $\mathrm{nd}^{3}$ & --- & --- & --- \\
\hline 24 & Palmeira/ PR & $\mathrm{Fl} / \mathrm{MG}$ & $\mathrm{Cg}$ & Sensível & 0,27 & 0,00 & 0,00 \\
\hline 25 & Palmeira/ PR & $\mathrm{Fl} / \mathrm{MG}$ & $\mathrm{Cg}$ & Mod. Sens. & 0,23 & 0,00 & 0,00 \\
\hline 26 & Palmeira/ PR & $\mathrm{Fl} / \mathrm{MG}$ & $\mathrm{Cg}$ & Sensível & 0,27 & 0,00 & 0,00 \\
\hline 27 & Palmeira/ PR & $\mathrm{Fl} / \mathrm{MG}$ & $\mathrm{Cg}$ & Sensível & 0,27 & 0,00 & 0,00 \\
\hline 28 & Palmeira/ PR & $\mathrm{Fl} / \mathrm{MG}$ & $\mathrm{Cg}$ & Sensível & 0,27 & 0,00 & 0,00 \\
\hline 29 & Palmeira/ PR & $\mathrm{Fl} / \mathrm{MG}$ & $\mathrm{Cg}$ & Mod. Sens. & 0,24 & 0,00 & 0,00 \\
\hline 30 & Palmeira/ PR & $\mathrm{Fl} / \mathrm{MG}$ & $\mathrm{Cg}$ & Sensível & 0,27 & 0,00 & 0,00 \\
\hline 31 & Fraiburgo & $\mathrm{Fl} / \mathrm{MN}$ & $\mathrm{Ca}$ & Resistente & 0,27 & 0,21 & 0,25 \\
\hline 32 & Fraiburgo & $\mathrm{Fl} / \mathrm{MN}$ & $\mathrm{Ca}$ & Resistente & 0,25 & 0,25 & 0,26 \\
\hline 33 & Fraiburgo & $\mathrm{Fl} / \mathrm{MN}$ & $\mathrm{Ca}$ & Resistente & 0,27 & 0,28 & 0,27 \\
\hline 34 & Fraiburgo & $\mathrm{Fl} / \mathrm{MN}$ & $\mathrm{Ca}$ & Resistente & 0,25 & 0,19 & 0,27 \\
\hline 35 & Palmas/ PR & $\mathrm{Fr} / \mathrm{PA}$ & $\mathrm{Ca}$ & Resistente & 0,27 & 0,27 & 0,27 \\
\hline 36 & Palmas/PR & $\mathrm{Fr} / \mathrm{PA}$ & $\mathrm{Ca}$ & Resistente & 0,27 & 0,22 & 0,24 \\
\hline 37 & Palmas/ PR & $\mathrm{Fr} / \mathrm{PA}$ & $\mathrm{Ca}$ & Resistente & 0,25 & 0,29 & 0,24 \\
\hline 38 & São Joaquim/ SC & $\mathrm{Fr} / \mathrm{PA}$ & $\mathrm{Ca}$ & Resistente & 0,30 & 0,29 & 0,28 \\
\hline 39 & São Joaquim/ SC & $\mathrm{Fr} / \mathrm{PA}$ & $\mathrm{Ca}$ & Resistente & 0,27 & 0,24 & 0,27 \\
\hline 40 & São Joaquim/ SC & $\mathrm{Fr} / \mathrm{PA}$ & $\mathrm{Ca}$ & Resistente & 0,30 & 0,27 & 0,30 \\
\hline
\end{tabular}

$1 \mathrm{Fl}=$ folha; $\mathrm{Fr}=$ fruto $; \mathrm{MG}=$ mancha da gala; $\mathrm{MN}=$ mancha necrótica; $\mathrm{PA}=$ podridão amarga

$2 \mathrm{Ca}=$ Colletotrichum acutatum $; \mathrm{Cg}=$ Colletotrichum gloeosporioides

$3 \mathrm{nd}=$ não determinado

fungicida ainda não é bem conhecida. Um terceiro grupo foi formado por quatro isolados de C. gloeosporioides $(10,26 \%)$ que apresentaram menor crescimento seis dias após a repicagem, quando submetidos a concentrações de benomyl (Figura 1), mas que não apresentaram IR\% diferente do padrão CG197.
Portanto, dentre os isolados de C. gloeosporioides examinados, foram identificados três grupos quando se levou em consideração a sensibilidade ao benomyl, um grupo com isolados sensíveis, um com isolados moderadamente sensíveis e outro com isolados insensíveis ao fungicida. A única diferença observada entre os isolados dos três grupos 


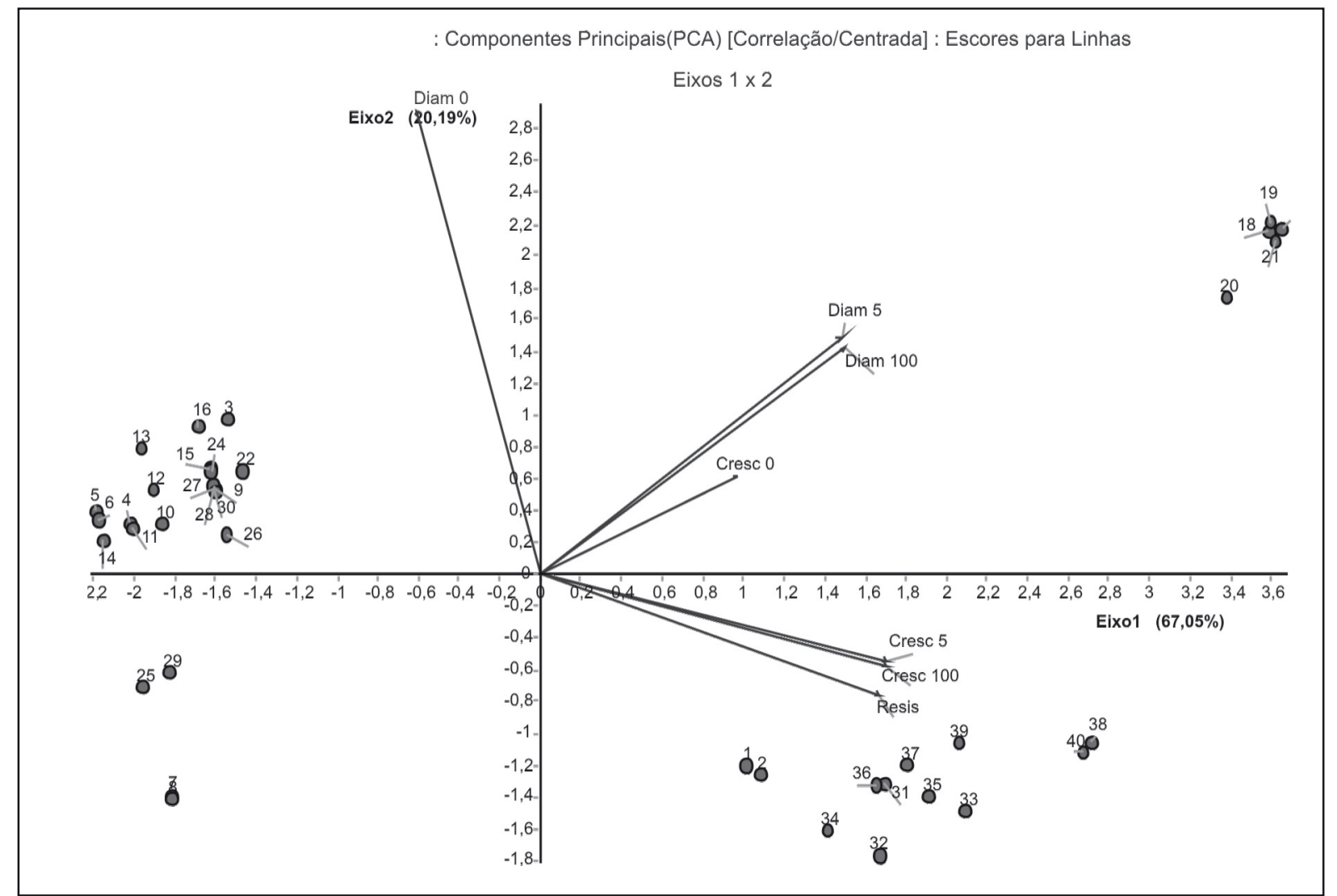

FIGURA 1 - Análise multivariada dos componentes principais (PCA) centrados e com os dados estandardizados, mostrando a dispersão das notas para Resistência (nota 1) ou Suscetibilidade (nota 0), do diâmetro (mm) das colônias de Colletotrichum spp. submetidas ao meio de cultura contendo zero (0), cinco (5) ou $100 \mu \mathrm{g} \cdot \mathrm{mL}^{-1}$ de benomyl, e suas respectivas taxas de crescimento.

de C. gloeosporioides foi à sensibilidade ao benomyl, pois estes não diferiram estatisticamente para as demais variáveis analisadas (coloração da colônia, produção de peritécios em meio de cultura, velocidade de crescimento em meio BDA, etc). Sendo assim, pode-se dizer que os isolados considerados insensíveis e/ ou moderadamente sensíveis são isolados de $C$. gloeosporioides que diferem dos até então estudados no patossistema Colletotrichum - maçã, no Brasil. Isolados identificados como C. gloeosporioides e que apresentaram resistência ao fungicida benomyl são relatados nos patossistemas Colletotrichum - maçã por Ishii et al. (1998) e Colletotrichum - mamão por Peres et al. (2002) e por Andrade et al. (2007). Segundo Delp (1980), os problemas de resistência de fungos ao grupo dos benzimidazóis intensificaram-se, não somente devido ao uso indiscrimado desses produtos nas lavouras, mas também pelo fato de eles atuarem em um sítio específico de metabolismo dos patógenos.

Diante destas observações, questiona-se a validade da utilização isolada do parâmetro "resistência ao fungicida benomyl" para a diferenciação entre $C$. gloeosporioides e C. acutatum em macieira. Sugere-se que este parâmetro seja associado a outras metodologias, tais como caracterização morfocultural, patogênica e até mesmo molecular, para uma identificação mais precisa, pois como já dito anteriormente, a correta identificação de espécies envolvidas em um patossistema é fundamental para um direcionamento adequado dos programas de melhoramento que visem a produção de plantas resistentes a doenças.

\section{REFERÊNCIAS BIBLIOGRÁFICAS}

Andrade EM, Uesugi CH, Ueno B, Ferreira MASV (2007) Caracterização morfocultural e molecular de isolados de Colletotrichum gloeosporioides patogênicos ao mamoeiro. Fitopatologia Brasileira 32:21-31.

Berstein B, Zehr EI, Dean RA, Shabi E (1995) Characteristics of Colletotrichum from peach, apple, pecan, and other hosts. Plant Disease 79:478-482.

Boneti JIS, Katsurayama Y, Bleicher J (1999a) Doenças da macieira (Malus domestica Bork) In: Boneti JIS, Ribeiro LG, Katsurayama Y (Eds.) Manual de identificação de doenças e pragas da macieira. Florianópolis SC. Empresa de Pesquisa Agropecuária e Extensão Rural de Santa Catarina - Epagri. 
Boneti JIS, Katsurayama Y, Bleicher J (2002) Doenças Fúngicas Foliares. A Cultura da Macieira. Florianópolis SC. Empresa de Pesquisa Agropecuária e Extensão Rural de Santa Catarina Epagri.

Boneti JIS, Katsurayama Y, Ozawa T (1999b) Levantamento das espécies de Colletotrichum associadas com a mancha foliar de Glomerella em macieira, cv. Gala, no Estado de Santa Catarina. Fitopatologia Brasileira 24:143. (Resumo)

Brown AE, Sreenivasaprasad S, Timer LW (1996) Molecular characterization of slow-growing orange and key lime anthracnose strains of Colletotrichum from citrus as C. acutatum. Phytopathology 86:523-527.

Chung W, Ishii H, Nishimura K, Fukaya M, Yano K, Kajitani Y (2006) Fungicide sensitivity and phylogenetic relationship of anthracnose fungi isolated from various fruit crops in Japan. Plant Disease 90:506-512.

Delp CJ (1980) Coping with resistance to plant disease control agents. Plant Disease 64:652-658.

Freeman S, Katan T, Shabi E (1998) Characterization of Colletotrichum species responsible for anthracnose diseases of various fruits. Plant Disease 82:596-605.

Goes A, Kimati H (1998) Colletotrichum acutatum, agente causal da queda prematura dos frutos cítricos: resistente ou insensível a benomyl? Summa Phytopathologica 24:246-253.

Ishii H, Iwamoto S, Nishimura K, Fukaya M (1998) Comparative studies on fungicide sensitivity and other characteristics in Colletotrichum isolated from various plant species. Proceedings, Brighton Crop Protection Conference: Pests and Diseases. Farnham UK. British Crop Protection Council. pp. 529-534.
Katsurayama Y, Boneti JIS, Ozawa T (2000) Sobrevivência de Colletotrichum gloeosporioides, agente causal da mancha foliar da gala. Fitopatologia Brasileira 25 (Supl):383.

Katsurayama Y, Boneti JIS, Becker WF, Ozawa T, Tsuchiya S (2001) Epidemiologia das Doenças da Macieira no Subtrópico e Perspectivas de Manejo Integrado: Caso da Mancha da Gala (Colletotrichum spp.). Anais, Seminário sobre fruticultura de clima temperado: Perspectivas da Fruticultura de Clima Temperado na Região Subtropical: Estratégias e Tecnologias para a Sustentabilidade da Fruticultura de Baixo Impacto Ambiental. Florianópolis SC. pp. 125-139.

Leite Jr RP, Tsuneta M, Kishino AY (1988) Ocorrência de mancha foliar de Glomerella em macieira no Estado do Paraná. Londrina PR. Instituto Agronômico do Paraná - IAPAR. Informe de Pesquisa, no. 81 .

Peres NAR, Kuramae EE, Dias MSC, Souza NL (2002) Identification and characterization of Colletotrichum spp. affecting fruit after harvest in Brazil. Journal of Phytopathology 150:128134.

Peres NAR, Souza NL, Peever TL, Timmer LW (2004) Benomyl sensitivity of isolates of Colletotrichum acutatum and $C$. gloeosporioides from citrus. Plant Disease 88:125-130.

Shabi E, Katan T, Gera H, Elisha S (1993) Taxonomic determination of pathogenic Colletotrichum gloeosporioides of almond, anemone and avocado according to fungicide sensitivity. Phytoparasitica 21:2. (Abstract)

Teixeira H, Chitarra LG, Sarias SMS, Machado JC (2001) Efeito de diferentes fontes de luz no crescimento e esporulação in vitro de fungos fitopatogênicos. Ciência e Agrotecnologia 25:1314-1320. 